\title{
Nordiques
}

$34 \mid 2017$

La mer Baltique comme zone-frontière : perspectives environnementales, géopolitiques, culturelles

\section{La Baltique orientale, périphérie stratégique de la sphère britannique de la guerre de Crimée à la première guerre mondiale, entre confrontation et coopération}

Philippe Blanc

\section{OpenEdition \\ Journals}

Édition électronique

URL : https://journals.openedition.org/nordiques/1653

DOI : 10.4000/nordiques. 1653

ISSN : 2777-8479

Éditeur :

Association Norden, Bibliothèque de Caen la mer

Édition imprimée

Date de publication : 1 novembre 2017

Pagination : 11-29

ISBN : 9791095914006

ISSN : $1761-7677$

\section{Référence électronique}

Philippe Blanc, «La Baltique orientale, périphérie stratégique de la sphère britannique de la guerre de Crimée à la première guerre mondiale, entre confrontation et coopération », Nordiques [En ligne], 34 | 2017, mis en ligne le 23 septembre 2021, consulté le 27 novembre 2021. URL : http://

journals.openedition.org/nordiques/1653; DOI : https://doi.org/10.4000/nordiques.1653 


\section{La Baltique orientale, périphérie stratégique de la sphère britannique de la guerre de Crimée à la première guerre mondiale, entre confrontation et coopération}

Philippe Blanc*

\section{RÉSUMÉ}

En 1856, la guerre de Crimée débouche, dans la Baltique, sur une forme de suprématie navale britannique, dont le pendant diplomatique est incarné par le traité d'Intégrité de novembre 1855, qui repousse un peu plus vers l'est la périphérie de la sphère stratégique britannique et sa "frontière " avec la sphère russe. C'est pour cela que le secteur finlandais, en particulier, va servir, dans les décennies qui suivent, d'espace de confrontation entre les deux puissances du Grand Jeu, davantage en fonction de la conjoncture internationale que de l'intérêt économique réel de la Baltique pour la Grande-Bretagne. Inversement, le jeu des nouvelles alliances et rivalités européennes du début du XXe siècle va faire de la Baltique orientale la caisse de résonance d'une nouvelle forme de coopération anglo-russe, l'attitude britannique influençant la donne locale, dans la coopération comme dans la confrontation.

\section{ABSTRACT}

In 1856, the Crimean War resulted in a form of British naval supremacy in the Baltic, whose diplomatic counterpart was embodied by the Integrity Treaty of November 1855, which pushed the periphery of the British strategic sphere and its "frontier" with the Russian sphere further to the East. That was the reason why the Finnish sector, in particular, acted, during the following decades, as a confrontation area between the two powers of the Great Game, depending more on international circumstances than on the actual economic interest of the Baltic zone for Britain. Conversely, through the new European alliances and rivalries that emerged in the early twentieth century, the Eastern Baltic came to reflect a new form of Anglo-Russian cooperation, as the British attitudes affected the local conditions, in cooperation as well as in confrontation.

* Philippe Blanc est PRAG d'anglais à l'université de Bourgogne Franche-Comté et docteur en histoire contemporaine. 


\section{INTRODUCTION}

Encore en 1905, la Suède, qui fait pourtant figure de pays le plus avancé de la Baltique, est considérée par les représentants diplomatiques britanniques comme se situant "à la périphérie de la géographie militaire et de la grande politique européennes " ${ }^{1}$. Plus généralement, durant tout le XIX ${ }^{e}$ siècle, la zone baltique, $\mathrm{du}$ moins dans sa partie orientale, est encore perçue dans les représentations des Britanniques qui s'y intéressent, à l'exemple des voyageurs, comme une périphérie sous-développée, à la frontière entre leur propre sphère culturelle occidentale et la sphère russe $e^{2}$.

Sur le plan économique, toutefois, il y a des raisons de penser que la relative importance de la Baltique dans son ensemble pour les Britanniques lui confère une place à part aux yeux du Foreign Office, même si cette importance paraît plus qualitative que quantitative. En effet, si le commerce avec la Baltique ne représente que $13 \%$ environ du commerce britannique en 1906 (en tonnage) ${ }^{3}$, la région dans son ensemble reste primordiale pour l'importation de certains produits indispensables, à l'instar du bois de construction (encore $57 \%$ des importations britanniques de bois non scié en 1910 , et $72 \%$ pour le bois scié) $)^{4}$.

Mais la question que l'on peut se poser est de savoir si la constante économique justifie un intérêt des Britanniques tout aussi continu pour cette zone périphérique de leur sphère stratégique. En effet, l'intérêt stratégique d'une région n'est pas seulement fonction de son éventuelle importance économique, en ce sens que cette région est également susceptible de subir des évolutions qui font écho à des problèmes internationaux plus lointains. C'est essentiellement à partir de ce rôle de " caisse de résonance " de la Baltique que nous voudrions montrer dans quelle mesure, parfois loin des préoccupations économiques locales, ces développements internationaux ont pu affecter sa partie orientale, qui constitue le point de

1 Wilhelm M. Carlgren, «Sweden, the Great Powers and the Baltic, 1890-1914 », in In Quest of Trade and Security. The Baltic in Power Politics, 1500-1990, vol. II (1890-1990), G. Rystad, K.-R. Böhme et W. M. Carlgren (dir.), Lund, Lund University Press, 1995, p. 22. Les passages originaux en anglais ont été traduits en français par l'auteur.

2 Philippe Blanc, La Grande-Bretagne, le Nord et la Baltique du milieu du XIXe siècle jusqu'à la Première Guerre mondiale. Des relations et un regard en évolution, thèse de doctorat en histoire contemporaine, Université de Strasbourg, 2016, 1 vol. (699 p.), p. 64-80.

3 Sources : pour la zone baltique, Archives nationales britanniques (The National Archives), HD 3/133, bilan des échanges commerciaux (valeurs et tonnages) entre le Royaume-Uni et la zone baltique en 1906, attaché au rapport secret du capitaine E. Slade (D.N.I.) à l'Amirauté du 25 novembre 1907 (British policy in the Baltic in the event of war with Germany); et pour les données globales : David J. Starkey (dir.), Shipping Movements in the Ports of the United Kingdom, 1871-1913. A Statistical Profile, Exeter, University of Exeter Press, 1999, p. 72-73.

4 Sources: House of Commons Parliamentary papers (HCPP), Annual statement of the trade of the United Kingdom with foreign countries and British possessions. 1910 compared with four preceding years. Compiled in the Statistical Office of the Customs and Excise Department. Supplement to vols. I and II. 1911 [Cd. 5699, 5852, 5853], LXXX.1, p. 210-215 (634-639). 
rencontre entre la sphère économique (et maritime) britannique et la sphère russe. Pour ce faire, nous avons choisi de nous concentrer sur une période suffisamment longue, de plus d'un demi-siècle, traversée par d'importants bouleversements, située entre les deux conflits qui voient la Grande-Bretagne intervenir militairement sur le continent européen entre le milieu du XIX ${ }^{\mathrm{e}}$ et le début du XX $\mathrm{XX}^{\mathrm{e}}$ siècle, à savoir la guerre de Crimée et la première guerre mondiale.

\section{La guerre de Crimée : la Baltique orientale au ceeur DE LA CONFRONTATION ANGLO-RUSSE}

Dès les années 1830, la crainte de l'expansionnisme russe, en particulier vers la route des Indes et le détroit des Dardanelles, mais aussi en Europe, donne lieu à une forme de "guerre froide anglo-russe ", appelée "le Grand Jeu " (Great Game $)^{6}$ entre les deux pays, alimentée également par l'opposition entre libéralisme et autocratie. Avec cette bipolarisation relative de l'Europe, les régions baltiques constituent forcément une ligne de fracture au cœur de l'antagonisme anglo-russe ${ }^{7}$, d'autant plus que dans les décennies qui suivent le traité de Fredrikshamn (Hamina) (1809), qui fait passer la Finlande sous son autorité, la Russie " développe un programme de mare clausum, pour exclure de l'espace baltique les navires de guerre des puissances non riveraines ${ }^{8}$. Selon l'Amirauté britannique, cela pourrait, à terme, fermer la mer Baltique à toute navigation britannique, militaire ou autre.

Outre les détroits danois, "bastions avancés de sa frontière baltique ", la ligne de défense russe principale se concrétise, dès les années 1830, par des fortifications sur les deux rives du golfe de Finlande, à la fois à Bomarsund (Åland), Sveaborg (Suomenlinna), Cronstadt, Reval (Tallinn) et à Dünamünde (Daugavgrïva), près de Riga, en vue de protéger l'accès à Saint-Pétersbourg. Bien entendu, entre les années 1830 et 1850 , les relations anglo-russes ne sont pas constamment sous tension, mais à l'été 1853 , quelques semaines avant le début des hostilités de la guerre de Crimée entre Russes et Ottomans, l'ambassadeur de Grande-Bretagne à Saint-Pétersbourg, George Hamilton Seymour, exprime son sentiment, selon lequel la Russie entrera tôt ou tard en "collision avec l'Europe occidentale ${ }^{10}$.

5 Andrew Lambert, " "This is all we want”, Great Britain and the Baltic Approaches 1815-1914 », in Britain and Denmark. Political, Economic and Cultural Relations in the $19^{\text {th }}$ and $20^{\text {th }}$ Centuries, J. Sevaldsen (dir.), Copenhague, Museum Tusculanum Press, 2003, p. 154.

6 Peter Hopkirk, The Great Game. On Secret Service in High Asia, Oxford, Oxford University Press, 2001 [1991], p. 1.

7 Carl Fredrik Palmstierna, "Sweden and the Russian Bogey ", The Nineteenth Century and After, vol. 113, 1933, p. 739-754 [réimpression].

8 Andrew Lambert, op. cit., p. 148-149.

9 Ibid., p. 153.

10 Muriel Chamberlain, " Pax Britannica " ? British Foreign Policy, 1789-1914, Londres, Longman, 1988, p. 106. 
Les opérations navales franco-britanniques dans la Baltique débutent au printemps 1854 et se résument assez vite à une stratégie de blocus maritime (entre Memel [Klaipeda] et Torneå [Tornio]), et à quelques actions sporadiques sur les côtes russes ; les éventuels projets d'attaques amphibies, sur Cronstadt en particulier, étant très vite abandonnés. Quoi qu'il en soit, c'est la partie orientale de la Baltique qui fait les frais du conflit armé anglo-russe dans un contexte où la flotte russe, largement inférieure à la première flotte de guerre à vapeur de l'histoire ${ }^{11}$, reste bien à l'abri de ses arsenaux. De fait, hormis quelques actions d'éclat individuelles, comme à Libau (Liepaja), le 16 mai 1854, et quelques bombardements de forteresses (Bomarsund, Sveaborg), ce sont les actions menées en Finlande qui, dans le cas présent, "matérialisent » la frontière militaire entre la sphère russe et celle dans laquelle la domination militaire britannique peut s'affirmer sans la moindre opposition, grâce à la déclaration de neutralité commune du Danemark et de la Suède-Norvège ${ }^{12}$.

Cette matérialisation de la ligne d'affrontement entre les deux sphères militaires, dans un contexte où, si l'on peut dire, un seul des belligérants se bat, fait d'ailleurs vite irruption de manière très brutale dans la vie des populations locales, comme les communautés côtières d'Ostrobotnie l'apprennent à leurs dépens. Alors que celles-ci n'ont strictement aucune valeur stratégique, ni militaire, elles se retrouvent en effet, à partir de l'été 1854 (mais pas seulement), sous le feu des lamentables attaques de l'amiral anglais Plumridge, dont le mot d'ordre de " capturer, brûler ou détruire » est sans équivoque ${ }^{13}$. Au final, non seulement ces exactions creusent-elles le fossé entre la sphère britannique et la sphère russe sur le plan géostratégique, mais aussi sur le plan culturel, car on saura, en Finlande, se souvenir de "l'amiral pyromane $»^{14}$.

C'est le traité de Paris du 30 mars 1856 qui met officiellement fin à la guerre de Crimée, mais ce sont d'autres évolutions baltiques simultanées qui affermissent la mainmise britannique sur cette mer, faisant par là même reculer la limite de leur zone d'influence un peu plus vers la partie orientale. C'est à l'initiative de Lord Palmerston que cette poussée se concrétise. Le Premier ministre (1855-1858) est en effet à l'origine du principal acte diplomatique du théâtre baltique de la guerre de Crimée, avec la signature, le 21 novembre 1855, du traité dit "d'Intégrité "

11 Basil Greenhill, Ann Giffard, The British Assault on Finland, 1854-1855. A Forgotten Naval War, Annapolis, Naval Institute Press, 1988, p. xi.

12 HCPP, Correspondence relative to the neutrality of Denmark, and Sweden and Norway, 1854 [1711], LXXII, p. 1-2 $(603,604)$; et The National Archives, FO 22/215, fo 1, lettre du comte ReventlowKriminil à Lord Clarendon du 2 janvier 1854.

13 Basil Greenhill, Ann Giffard, op. cit., p. 171.

14 Juhani Paasivirta, Finland and Europe. International Crises during the Period of Autonomy, 1808-1914, Londres-Minneapolis, University of Minnesota Press, 1981, p. 96-97. 
signé entre la France, la Grande-Bretagne et les royaumes de Suède-Norvège ${ }^{15}$, dans lequel ces derniers s'engagent "à ne céder à la Russie, ni à échanger avec elle, ni à lui permettre d'occuper, aucune partie des territoires appartenant aux Couronnes de Suède et de Norvège " - le Finnmark, en l'occurrence - en échange d'une protection franco-anglaise, navale et militaire, contre la Russie ${ }^{16}$. Au-delà de la simple expression d'un droit de regard et d'intervention franco-britannique, même protecteur, sur les affaires de la péninsule scandinave ${ }^{17}$, ce traité a pour but final, selon Lord Palmerston, de créer " une longue ligne de circonvallation destinée à contenir l'extension future de la Russie [...] [qui] [...] sera pour nous le meilleur moyen d'éviter toute future collision ${ }^{18}$. Commençant dans le Finnmark, que l'on considère à présent sécurisé pour la sphère occidentale, la "frontière " entre les deux zones, véritable cordon sanitaire, passe ensuite par le golfe de Botnie, à l'entrée duquel les escadres alliées ont accompli un des rares faits d'armes des opérations baltiques en détruisant la forteresse de Bomarsund (août 1854), sur les îles Åland, dont la convention du même nom, attachée au traité de Paris, obtient la démilitarisation.

Cette nouvelle frontière maritime, plus orientale, place tout naturellement la Finlande définitivement dans l'orbite russe ${ }^{19}$, si l'on avait pu douter qu'elle en fit partie, même si on trouve, pendant la guerre, quelques journaux britanniques (le Standard et le Daily News) pour croire encore que la Grande-Bretagne pourrait aider la Suède à récupérer la Finlande ${ }^{20}$. D'ailleurs, par un effet miroir, la vision finlandaise des Occidentaux, et surtout des Anglais, n'évoque pas précisément des idéaux de liberté et de progrès, mais plutôt un sentiment de révulsion face à une puissance maritime brutale, qui recherche l'hégémonie à tout prix ${ }^{21}$.

\section{Le Grand Jeu et la Baltique après la guerre de Crimée}

L'historien naval britannique, Andrew Lambert, considère la mer Baltique comme « le seul théâtre qui représente un intérêt vital [pour la Grande-Bretagne] pendant tout le XIX ${ }^{e}$ siècle $»^{22}$. Cette affirmation peut faire l'objet de nombreux débats, mais, si elle contient une grande part de vérité, il convient d'en moduler la portée comme le montre un événement assez révélateur.

15 Paul Knaplund, "Finmark in British Diplomacy, 1836-1855 ", American Historical Review, vol. 30, no 3, avril 1925, note de bas de page no 84 , p. 497 et p. 498 .

16 FO 94/470, traité d'Intégrité du 21 novembre 1855 (en français dans le texte).

17 Paul Knaplund, op. cit., p. 499.

18 FO 73/269, cité dans ibid.

19 Juhani Paasivirta, op. cit., p. 104.

20 George Maude, Finland and Britain. 1854-1914, thèse Ph.D., University of London, 1970, 1 vol. (377 p.), p. 27, et Juhani Paasivirta, op. cit., p. 93.

21 Ibid., p. 96-97.

22 Andrew Lambert, "Great Britain and the Baltic, 1890-1914 ", in Britain and the Baltic. Studies in Commercial, Political and Cultural Relations, 1500-2000, P. Salmon et T. Barrow (dir.), Sunderland, University of Sunderland, 2003, p. 216. 
En effet, lors de la seconde guerre du Schleswig ${ }^{23}$, qui voit, en février 1864, les troupes prussiennes (et autrichiennes) envahir ce duché, puis le Danemark proprement dit (Jutland), Londres n'entreprend aucune action diplomatique sérieuse, et encore moins militaire, alors même que les détroits sont menacés. Cela constituait pourtant, jusqu'alors, un casus belli pour la Grande-Bretagne, mais au-delà, la principale crainte du gouvernement de Londres était, comme le résume le sous-secrétaire d'État aux Affaires étrangères, Henry Layard, que « la guerre, allumée dans le Schleswig, n’embrasât tout le continent $»^{24}$, ce qui eût été fort préjudiciable aux intérêts économiques britanniques.

On mesure, en cette occasion, la remarquable capacité d'adaptation du gouvernement britannique, s'agissant d'une zone que l'on considère comme plus ou moins sécurisée à moyen terme. C'est d'ailleurs Lord Palmerston lui-même ${ }^{25}$ qui, parlant de la convention de Gastein en $1865^{26}$, résume l'idée générale : «Je dirais qu'il vaut mieux que [le Schleswig et le Holstein] aillent augmenter la puissance de la Prusse plutôt que de former un autre petit État [...] afin de contrôler ces deux puissances ambitieuses et agressives que sont la France et la Russie... $»^{27}$. Finalement, cela suffit bien pour une Grande-Bretagne qui se désintéresse peu à peu du continent européen : la zone baltique ne perd rien de son intérêt économique ${ }^{28}$, mais ne nécessite plus vraiment d'être considérée comme un objectif stratégique majeur en soi.

Malgré tout, la guerre de Crimée a montré que la Baltique risquait surtout de subir le feu britannique lorsqu'il s'agissait d'enjeux encore plus importants pour la Grande-Bretagne, mais aussi plus lointains, en Méditerranée orientale notamment. Or, l'antagonisme anglo-russe du Grand Jeu n'est toujours pas terminé, mais il a pour cadre essentiel le Moyen-Orient, sur la ligne qui sépare les possessions britanniques de l'Empire russe (Perse, Afghanistan et sous-continent indien), et dans la région des Balkans et de la mer Noire, gardienne du verrou des Dardanelles. C'est surtout dans ce cadre-là que l'on continue, à Londres, de surveiller la Baltique avec un "détachement attentif ${ }^{29}$.

23 Sur l'attitude de la Grande-Bretagne durant la guerre des duchés, lire Keith Sandiford, Great Britain and the Schleswig-Holstein Question, 1848-1864. A Study in Diplomacy, Politics and Public Opinion, Toronto, University of Toronto Press, 1975.

24 Émile Pirazzi, L'Angleterre et l'Allemagne. À propos du Schleswig-Holstein, Bruxelles-Leipzig-Paris, 1865 , p. 127.

25 Lord Palmerston est alors de nouveau Premier ministre (1859-1865).

26 La convention de Gastein (14 août 1865) décide que le Schleswig sera administré par la Prusse et le Holstein par l'Autriche.

27 Lettre privée de Lord Palmerston à Lord Russell du 13 septembre 1865, in The Foreign Policy of Victorian England. 1830-1902, K. Bourne (dir.), Oxford, Clarendon Press, 1970, p. 381-382.

28 La valeur réelle des marchandises importées et exportées entre le Royaume-Uni et la Baltique passe de 30 millions de livres au milieu du XIX ${ }^{\mathrm{e}}$ siècle à 47 millions de livres en 1865 (Keith Sandiford, op. cit., p. 33).

29 Ibid., p. 139. 
Mais c'est dans les années 1870 que les choses se gâtent, et les événements qui affectent le Proche-Orient en 1876 ne sont pas sans rappeler les " préliminaires de la guerre de Crimée ${ }^{30}$. Lorsqu’en avril 1877, la Russie déclare la guerre à la Turquie, la Grande-Bretagne reste neutre dans ce conflit, qui génère tout de même des tensions car celui-ci risque d'affecter Constantinople, porte des Dardanelles. Or, précisément, le traité de San Stefano (mars 1878), qui crée la Grande Bulgarie $^{31}$, provoque le refus britannique exposé dans la " circulaire de Lord Salisbury " du $1^{\text {er }}$ avril 1878, arguant du fait qu'un contrôle des détroits exercé par la seule Russie « constituerait un remède illusoire aux dangers posés aux intérêts anglais et à la permanence de la paix en Europe $»^{32}$.

Sept ans plus tard, on assiste de nouveau à un regain de tension au printemps 1885, toujours dans le cadre du Grand Jeu, lorsque les Russes tentent de s'avancer en territoire afghan, présentant une nouvelle menace sur un secteur sensible, qui sert de zone tampon, sous influence britannique, entre l'Empire russe et les possessions indiennes de la Grande-Bretagne.

Comme lors de la guerre de Crimée, c'est assez logiquement qu'en 1878, puis en 1885, la mer Baltique se retrouve de nouveau affectée par des tensions concernant un autre endroit du globe. Et en 1885 comme en $1878^{33}$, on assemble donc en Grande-Bretagne une flotte baltique. La " frontière " baltique se trouve ainsi réactivée, d'autant plus qu'au cours des deux crises, les pays scandinaves, notamment le Danemark, ne semblent pas vouloir faire obstacle au libre passage de navires de guerre britanniques ${ }^{34}$.

À l'échelle locale, l'emploi d'une éventuelle flotte baltique britannique pouvant faire pression sur la Russie implique une surveillance accrue de la zone orientale aux moments de tension, montrant par là que la frontière entre le champ d'action britannique et les eaux russes a une existence tout à fait concrète. Au printemps 1878 en particulier, au sommet de la crise russo-turque, c'est bien sûr le point névralgique de Cronstadt qui fait l'objet de toutes les attentions. C'est la raison pour laquelle le colonel Wellesley, attaché militaire à l'ambassade de SaintPétersbourg, demande un rapport sur la faisabilité d'une attaque sur Cronstadt par le nord, à partir d'une tête de pont terrestre ${ }^{35}$.

30 Muriel Chamberlain, op. cit., p. 139.

31 Ibid., p. 139-140.

32 FO 244/314/190, circulaire de Lord Salisbury du $1^{\text {er }}$ avril 1878, in K. Bourne (dir.), op. cit., p. 412 .

33 Andrew Lambert, " "This is all we want”... », op. cit., p. 160.

34 Carsten Due-Nielsen, "The Beginning of a Beautiful Friendship : Denmark's Relations with Britain, 1864-1914 ", in J. Sevaldsen (dir.), op. cit., p. 178.

35 FO 65/1004, dépêche secrète de l'ambassadeur Lord A. Loftus (Saint-Pétersbourg) à Lord Salisbury du 5 juin 1878 . 
En réponse à cette demande, le plan établi par un certain capitaine A. Noedt conclut que le point idéal de débarquement se situe à quelques kilomètres au nord du village de Sestroretsk, en face de l'île de Cronstadt (dix kilomètres au nord-est), car celui-ci possède « une plage en pente douce, formant une crique [...] avec l'ancrage le plus sûr que l'on puisse imaginer », où « les navires de guerre d'eau profonde les plus puissants [...] peuvent approcher de la plage en toute sécurité à moins d'un demi-mile $»^{36}$.

Toutefois, cette dimension stratégique de la Baltique orientale fait également de la Finlande une zone à surveiller en tant que territoire russe. D'ailleurs, dans les années 1870, « les relations avec la Finlande ne sont jugées qu’à travers le prisme de son importance stratégique ${ }^{37}$, ou, plus précisément, comme "le fragile flanc maritime occidental » du colosse russe ${ }^{38}$. À vrai dire, ce n'est qu'aux moments de tensions cités ci-dessus que l'intérêt stratégique de la Finlande resurgit. Ainsi, une fois la guerre russo-turque enclenchée, les Finlandais, craignant une répétition de la guerre de Crimée, entreprennent, au printemps 1878, des travaux de fortification et de préparation sur la côte méridionale du pays ${ }^{39}$, au sujet desquels le personnel diplomatique envoie au Foreign Office des informations qui montrent que l'éventualité d'une action militaire à cet endroit n'est pas dédaignée de part et d'autre. Dans le secteur finlandais, par exemple, qui surveille le couloir d'entrée vers Saint-Pétersbourg, on apprend que :

une division d'infanterie [a été] formée à Sveaborg "pour la défense de la forteresse » [...]. « Des postes de surveillance ont été établis le long de la côte finlandaise entre la passe de Björkö ${ }^{40}$ et Saint-Pétersbourg [, et] une nouvelle route militaire est en construction » depuis trente kilomètres à l'est de Wyborg (Viipuri) jusqu’à Björkö.. ${ }^{41}$

De même, à l'automne 1884, le Foreign Office demande encore à William Campbell, consul à Helsingfors (Helsinki), de lui fournir des dessins précis de la forteresse de Sveaborg ${ }^{42}$.

36 FO 65/1004, rapport du capitaine A. Noedt du 5 juin 1878 (« Materials for an operation against Cronstadt ").

37 George Maude, op. cit., p. 78.

38 Ibid., p. 50 et 87.

39 Juhani Paasivirta, op. cit., p. 155-156.

40 Il s'agit des Îles Beriozovye (Koiviston Saaret en finnois), situées entre Vyborg et Saint-Pétersbourg.

41 FO 65/1004, mémorandum du consul John Michell (Saint-Pétersbourg) du 4 juin 1878.

42 FO 65/1193/5 (15 septembre 1884), cité par George Maude, op. cit., p. 91. 
Ainsi donc, si la mer Baltique n'est plus, à proprement parler, un enjeu stratégique aussi vital que la mer Noire - et ce, même au début du $\mathrm{XX}^{\mathrm{e}}$ siècle ${ }^{43}$ - elle reste une avenue "par laquelle peuvent être attaquées des puissances terrestres hostiles mais hors d'atteinte autrement $"^{44}$.

De fait, à cause de ce rôle assigné à la Baltique, semble se faire jour, en Finlande en particulier, une forme de "syndrome frontalier » propre aux tensions anglorusses. Lors de la crise afghane de 1885, par exemple, les Finlandais ne se font aucune illusion sur le statut de terre russe de leur pays aux yeux des Britanniques. Et, se souvenant des dégâts causés par les Anglais pendant la guerre de Crimée, certains demandent aux représentants britanniques à Saint-Pétersbourg, à l'instar du quotidien libéral svécophone Helsingfors Dagblad, de respecter la neutralité du grand-duché, tant on est conscient de se trouver sur un terrain de jeu entre grandes puissances ${ }^{45}$. Le consul général à Christiania, Thomas Michell, croit d'ailleurs savoir que les Finlandais ont exprimé le désir qu'en cas de guerre, les Anglais les considèrent autant que possible comme Finlandais (et non pas Russes) ${ }^{46}$.

\section{LE TOURNANT DE L'ENTENTE ANGLO-RUSSE DE 1907}

Dans la mesure où la Baltique semble être une caisse de résonance des soubresauts qui agitent les relations internationales, au moins entre la GrandeBretagne et la Russie, la région ne peut manquer d'être affectée par tout changement majeur intervenant dans le positionnement des deux pays. La première décennie du XXe siècle est décisive en la matière. Tout d'abord, la défaite navale russe de Tsushima, face aux Japonais, en mai 1905, semble élimer, pour un temps au moins, les dents de l'ours russe sur les théâtres d'opérations maritimes. En outre, le jeu des alliances qui se dessinent au tournant du siècle modifie également la donne baltique, puisque à partir de 1891-1894, l'alliance franco-russe réduit sensiblement la portée du traité d'Intégrité de $1855^{47}$. Celui-ci se retrouve même

43 Pour se donner une idée de la place de la Baltique dans l'échelle des zones à risques, il suffit de lire le mémorandum du Foreign Office sur les relations anglo-russes entre 1892 et 1904, qui explique en introduction que " les points de contact entre les gouvernements de le Grande-Bretagne et de la Russie sont principalement confinés à l'Asie ", ainsi qu’aux Dardanelles et à l'Égypte. La Baltique, quant à elle, n'est même pas mentionnée une seule fois en vingt et une pages (FO 881/8338, Memorandum respecting the relations between Russia and Great Britain, 1892-1904 [mémorandum confidentiel rédigé par J.A.C. Tilley le 14 janvier 1905]).

44 David Sweet, "The Baltic in British Diplomacy before the First World War ", The Historical Journal, vol. 13, no 3, 1970, p. 451.

45 George Maude, «Finland in Anglo-Russian Diplomatic Relations, 1899-1910 », The Slavonic and East European Review, vol. 48, no 113, 1970, p. 557 ; Juhani Paasivirta, op. cit., p. 156 ; et Pertti Luntinen, " Neutrality in Northern Europe before the First World War ", in Neutrality in History, J. Nevakivi (dir.), Helsinki, SHS/FHS, 1993, p. 108.

46 Dépêche no 9 du consul-général T. Michell (Christiania) du 12 avril 1885, citée dans FO 881/5906, mémorandum de F.H.T Streatfield du 26 février 1890 concernant le Varangerfjord.

47 Wilhelm M. Carlgren, op. cit., p. 10. 
quasiment condamné lorsque au tournant du siècle se profilent une entente anglo-russe et un renversement des priorités stratégiques britanniques en Europe.

Avant Tsushima, et avant la crise marocaine de Tanger la même année, le gouvernement d'Arthur Balfour (1902-1905) considère encore que l'ennemi, c'est la Russie, car les « unionistes continuent de penser en termes d'empire, plutôt qu'en termes d'équilibre des forces en Europe $"^{48}$. C'est bien pourtant à cette époquelà que se produit le tournant décisif, sous l'action des organismes nouvellement créés, chargés de planifier la future politique militaire de la Grande-Bretagne, à savoir le Committee of Imperial Defence (CID, 1902) et l'état-major (General Staff, 1904), ce dernier agissant au sein du War Office.

En fait, peut-être aussi dans une sorte de lutte d'influence de ces différents services, le War Office attaque en 1904 la suprématie de l'Inde dans la vision stratégique impériale du CID, créature d'Arthur Balfour. En effet, face à un potentiel de "troupes terrestres russes presque illimité ", le General Staff finit par conclure à "l'impossibilité de gagner une guerre contre la Russie " du côté de l'Inde ${ }^{49}$. À l'inverse, on en arrive vite, au sein de cet organe, et ce dès 1904, à parler d'une "guerre anglo-allemande inévitable ${ }^{50}$, dans un contexte de plus en plus tendu de rivalité navale et commerciale.

On assiste donc à un recentrage plus européen de la stratégie britannique, rendu possible par la consolidation de l'Entente cordiale avec la France, et par la convention anglo-russe du 31 août 1907. Compte tenu des priorités russes de la Grande-Bretagne, cet accord porte sur les questions « en rapport avec la Perse, l'Afghanistan, et le Tibet " ${ }^{51}$; et il change véritablement la donne entre les deux pays de par le relatif succès qu'il va connaître, avec des hauts et des bas. Au demeurant, l'ambiance à Londres vire assez vite à l'optimisme, comme l'écrit, dès novembre 1907, Charles Hardinge ${ }^{52}$, sous-secrétaire d'État permanent au Foreign Office (1906-1910), à son successeur à Saint-Pétersbourg, Arthur Nicolson (de 1906 à 1910) : " Heureusement, un risque de guerre avec la Russie a désormais disparu. ${ }^{53}$ Ce dernier considère d'ailleurs que " [sans la convention] il aurait

48 Joseph McDermott, "The Revolution in British Military Thinking from the Boer War to the Moroccan Crisis ", in The War Plans of the Great Powers, P. Kennedy (dir.), Londres, George Allen \& Unwin, p. 107.

49 Ibid., p. 105, 106 et 108.

50 Arthur J. Marder, From the Dreadnought to Scapa Flow. The Royal Navy in the Fisher Era, 1904-1919, vol. I, Londres, Oxford University Press, 1961, p. 110.

51 "Convention between the United Kingdom and Russia relating to Persia, Afghanistan, and Thibet ", in British Documents on the Origins of the War. 1898-1914, vol. IV, The Anglo-Russian Rapprochement. 1903-1907, G.P. Gooch et H. Temperley (dir.), Londres, 1929, p. 618-629, Annexe 1.

52 Secrétaire d'ambassade à Saint-Pétersbourg de 1898 à 1904, puis ambassadeur de 1904 à 1906.

53 Lettre privée de C. Hardinge à A. Nicolson du 12 novembre 1907, in British Documents on the Origins of the War. 1898-1914, vol. VIII, Arbitration, Neutrality and Security, doc. no 111, G.P. Gooch et H. Temperley (dir.), Londres, 1929, p. 136. 
fallu une baguette de magicien pour balayer, dans un délai si court, les nuages de suspicion, de rivalité et de défiance qui, pendant tellement de générations, ont obscurci les relations entre les deux pays $~^{54}$. Il est vrai que l'ambassadeur est luimême l'un des artisans du rapprochement anglo-russe, qui constitue pour lui le "socle» de la politique étrangère britannique ${ }^{55}$.

Mais pour ce qui nous intéresse ici, la question qu'il faut se poser est de savoir ce que devient, après ce recentrage de la stratégie britannique, et ce bouleversement des rivalités, la " ligne de circonvallation » issue du traité de 1855 ?

Bien entendu, concernant la Baltique, les priorités stratégiques britanniques et l'entente anglo-russe ne sont pas les seuls facteurs affectant les relations diplomatiques entre les deux pays dans la région ; tout juste en constituent-elles la toile de fond. Malgré le désastre de la flotte russe au Moyen-Orient, on n’a jamais oublié, à Saint-Pétersbourg, que la mer Baltique fut, à partir de la fin du stormaktstiden suédois (1721), considérée comme une mare russicum, dont pouvait être exclue en particulier la Grande-Bretagne, en partie au moyen de la ceinture de fortifications des années 1820-1830, évoquée précédemment ${ }^{56}$. Or, après 1905, la dissolution de l'union entre la Norvège et la Suède semble être l'occasion pour Alexandre Izvolski, ministre russe des Affaires étrangères, d'augmenter l'influence de son pays dans la région. Son projet comporte, entre autres, un rapprochement avec l'Allemagne en vue de surveiller les détroits danois pour exclure de la Baltique tout navire d'un pays non riverain - Grande-Bretagne comprise -, et surtout la suppression de la « servitude » des îles Åland, annexée au traité de Paris de 1856, afin de pouvoir remilitariser l'archipel ${ }^{57}$.

Le nouvel esprit régissant les rapports anglo-russes dans la Baltique se manifeste d'abord dans les réactions britanniques aux gestes de la Russie, qui auraient eu de quoi surprendre à une autre époque ; preuve qu'il y a du changement dans l'air. Ainsi, à cause d'une indiscrétion malheureuse de la part d'Alexandre

54 Rapport annuel pour 1908, envoyé par A. Nicolson à E. Grey le 8 février 1909, in British Documents on Foreign Affairs, partie 1, série A, vol. V, Russia. 1907-1909, doc. nº 61, K. Bourne et D. Cameron Watt (dir.), Frederick, University Publications of America, p. 215-216.

55 Eino Lyytinen, Finland in British Politics in the First World War, Helsinki, Suomalainen Tiedeakatemia, 1980, p. 45.

56 Jusqu’à la guerre de Crimée, l'influence et la pression russes sont également très importantes sur le plan politique dans les deux pays qui surveillent l'entrée de la Baltique, à savoir la Suède et le Danemark, puisque, par exemple, peu de décisions politiques danoises se prennent sans l'aval de Saint-Pétersbourg, y compris un simple traité commercial avec le Brésil (David Kirby, The Baltic World, 1772-1993. Europes Northern Periphery in an Age of Change, Londres - New York, Routledge, 1995, p. 97).

57 Wilhelm M. Carlgren, op. cit., p. 23-24, et protocole secret annexé au mémorandum d'Heinrich L. von Tschirschky du 7 août 1907. Joint au doc. no 106, in G.P. Gooch et H. Temperley (dir.), vol. VIII, op. cit., p. 132. 
Izvolski lui-même auprès d'Arthur Nicolson ${ }^{58}$, et d'une autre, plus volontaire, de l'ambassadeur allemand à Londres, le comte Metternich, auprès d'Edward Grey ${ }^{59}$, le 4 décembre $1907^{60}$, le Foreign Office se retrouve très vite informé du projet germano-russe de traité sur la Baltique, finalisé fin octobre, ayant pour objet «le maintien du statu quo territorial actuel sur la base de l'exclusion complète des affaires de la mer Baltique de toute influence politique étrangère ${ }^{61}$.

Alors même que l'on soupçonne, à Londres, une manœuvre diplomatique secrète visant à fermer les détroits danois en cas de guerre, loin de s'offusquer de la duplicité du ministre russe quelques mois après la signature de la convention anglo-russe, la réaction britannique reste étonnamment flegmatique. Pour preuve, Edward Grey précise à l'ambassadeur de France, Paul Cambon, qu’il « [ne voit] rien à quoi [la France et la Grande-Bretagne] pourr[aient] objecter dans l'arrangement qui est proposé entre la Russie, l'Allemagne et la Suède si [...] cela était [seulement] pour maintenir le statu quo et n'avait rien à voir avec l'idée de fermer ou de neutraliser la Baltique $»^{62}$. Cette réaction confirme d'une part la valeur stratégique, réelle mais relative à présent, de la mer Baltique pour le Foreign Office, qui se centre essentiellement sur la permanence de l'accès aux détroits danois, surtout pour la navigation commerciale, et d'autre part un souci manifeste de rester modéré face à une initiative russe quelque peu douteuse.

L'autre projet du ministre russe est donc de profiter de l'affaiblissement de la Suède après la dissolution de l'union, pour remettre en cause la convention de 1856, dont la Grande-Bretagne et la France sont signataires, sur l'interdiction faite aux Russes de fortifier et de militariser l'archipel des Åland. On appuie la requête en mettant en avant un souci très concret, à savoir qu'il devient nécessaire de contrôler la contrebande d'armes passant par la Finlande à destination des révolutionnaires russes ${ }^{63}$. Nouveau témoignage de la nouvelle atmosphère, plus bienveillante, qui semble prévaloir, contrairement à l'époque de la guerre de Crimée : dans un premier temps, le Foreign Office ne considère pas la demande russe comme étant illégitime dans un but de lutte contre la contrebande, pas plus

58 Communication de l'ambassadeur A. Nicolson à E. Grey du 19 novembre 1907, citée par David W. Sweet, op. cit., p. 468.

59 Ministre des Affaires étrangères (Foreign Secretary) de 1905 à 1916.

60 The National Archives, CAB 37/91/4, dépêche «très confidentielle » d'E. Grey à Lord Salisbury du 4 décembre 1907.

61 Protocole secret annexé au mémorandum d'Heinrich L. von Tschirschky du 7 août 1907, in G.P. Gooch et H. Temperley (dir.), vol. VIII, op. cit., p. 132.

62 FO 371/338, dépêche no 698 d'E. Grey à Mr E. Lister (fonctionnaire au Foreign Office de 1899 à 1907) du 9 décembre 1907, doc. no 117 , in ibid., p. 141.

63 J.O. Söderhjelm, Démilitarisation et neutralisation des îles d'Åland en 1856 et 1921, thèse de doctorat en droit, Université d'Helsingfors, 1928, 1 vol., p. 109. 
qu'il n'estime qu'elle représente un réel danger pour la tranquillité de la région ${ }^{64}$. Preuve, d'ailleurs, du peu de cas que l'on fait de cette affaire à Londres, avant même le projet russe sur Åland et la signature de l'Entente, le même Foreign Office oppose une fin de non-recevoir aux demandes des compagnies d'assurance maritime, comme la United Kingdom Mutual Steamship Assurance Association, qui craint d'éventuelles pertes commerciales causées par des inspections trop poussées de la marine de guerre russe dans le secteur des îles Åland et de la Finlande (été 1906) ${ }^{65}$.

Toutefois, en Suède, les visées de Saint-Pétersbourg ont pour effet, assez logiquement, de provoquer un vent de panique quant aux intentions réelles de l'ennemi héréditaire, compte tenu de la proximité de l'archipel avec Stockholm. Après réflexion, vu depuis Londres, on réalise assez vite qu'en effet, la demande russe arrive à un très mauvais moment, et ne peut que renforcer l'envie de la Suède, si la servitude d'Åland venait à être supprimée, de se tourner vers l'Allemagne, le nouvel adversaire ${ }^{66}$. La diplomatie britannique se retrouve donc obligée de louvoyer entre ses différents " amis ». On essaie donc d'une part de persuader le gouvernement russe d'offrir à la Suède suffisamment de garanties, mais, d'autre part, d'après Charles Hardinge, on se garde bien de montrer un soutien trop visible aux Suédois afin de ne pas "les encourager à s'entêter " ${ }^{67}$, et de ne pas faire trop obstacle aux désirs russes.

Début mars 1908, devant le tollé suscité par sa demande, Alexandre Izvolski jette l'éponge et semble abandonner le projet Åland ${ }^{68}$. Selon Folke Lindberg (1958) et Pertti Luntinen (1975), si le ministre russe cède si facilement, c'est aussi parce qu'il est soucieux de s'assurer « le soutien de la Grande-Bretagne contre les Empires germaniques " ${ }^{69}$ dans sa défense des intérêts des Slaves des Balkans contre l'Autriche-Hongrie (1908-1909). Là aussi, il s'agit donc de coopérer avec l'ancien ennemi sur des sujets autrement plus essentiels.

$64 \mathrm{CAB}$ 17/59, note confidentielle du 18 juillet 1906, envoyée à C. Hardinge (Foreign Office) (auteur inconnu).

65 FO 368/45/25599, lettre de la United Kingdom Mutual Steamship Assurance Association au Foreign Office du 26 juillet 1906, et FO 368/45/29755, lettre du Foreign Office à la United Kingdom Mutual Steamship Assurance Association du 12 septembre 1906.

66 Communication de l'ambassadeur F. Bertie (Paris) à E. Grey du 9 juillet 1907, citée par David W. Sweet, op. cit., p. 465.

67 Lettre privée de C. Hardinge à A. Nicolson du 3 mars 1908, doc. no 149, in G.P. Gooch et H. Temperley (dir.), vol. VIII, op. cit., p. 171.

68 FO 371/528, dépêche secrète du ministre britannique J. Rennell Rodd (Stockholm) à E. Grey du 3 mars 1908, doc. $\mathrm{n}^{\circ}$ 148, in ibid., p. 169-170 ; et Pertti Luntinen, The Baltic Question. 1903-1908, Helsinki, Suomalainen Tiedeakatemia, 1975, p. 223.

69 Pertti Luntinen, ibid., p. 224 ; et Folke Lindberg, Scandinavia in Great Power Politics. 1905-1908, Stockholm, Almquist \& Wiksell, 1958, p. 227. 
Cela n'empêche pourtant pas Alexandre Izvolski de remettre la question d'Åland sur le tapis en février de l'année suivante, prenant ainsi totalement à contrepied les craintes britanniques ${ }^{70}$, ce qui a bien sûr pour effet de terriblement embarrasser le Foreign Office, obligé de ménager la chèvre et le chou, la Russie et la Suède. C'est que les priorités sont claires pour Edward Grey, qui écrit à son ambassadeur à Stockholm, Cecil Spring Rice (1908-1913), que « nous [sommes] en bons termes avec la Russie ", et qu'il s'agit de le rester ${ }^{71}$. Il ne faut surtout pas froisser les nouveaux amis ${ }^{72}$, ce qui n'empêche pas le chef du Foreign Office d'être extrêmement irrité contre son homologue russe qu'il accuse d'avoir " commis une grosse faute en soulevant de nouveau la question des îles Åland, [car] cela a naturellement prédisposé le gouvernement suédois à se reposer sur l'Allemagne $»^{73}$. Néanmoins, au final, la diplomatie britannique se révèle plutôt efficace puisque la question de la remilitarisation de l'archipel est de nouveau abandonnée (jusqu'à la première guerre mondiale), avec une forme de coopération diplomatique dans le sens Saint-Pétersbourg - Londres, cette fois, dans la mesure où Alexandre Izvolski est, lui aussi, fortement impliqué dans l'entente anglo-russe ; question de priorité.

Au-delà de l'archipel des Åland, la compréhension britannique pour le partenaire russe se manifeste également, d'une manière passive pourrait-on dire, en Finlande même, dont le statut, aux yeux de certains Britanniques informés, passe de plus en plus - en termes socioculturels - de celui de zone russe à part entière, au moment du traité de 1855, à celui de zone intermédiaire entre Europe occidentale et Empire russe. Cette évolution est rendue particulièrement évidente par les comptes rendus qu'en font une poignée de voyageurs, souvent sympathisants socialistes, comme Mrs Alec Tweedie $(1897)^{74}$, Alexander McCallum-Scott $(1908)^{75}$ ou Rosalind Travers $(1911)^{76}$. D'après eux, la Finlande est sur la voie d'une modernisation spectaculaire, qui la rapproche des pays occidentaux, tout en l'éloignant de l'Empire russe proprement dit, et de son régime autocratique, aux antipodes des aspirations démocratiques finlandaises ${ }^{77}$.

70 FO 371/745, dépêche de C. Spring Rice à E. Grey du 17 août 1909, citée par David W. Sweet, op. cit., p. 479.

71 FO 371/745/29346, dépêche confidentielle d'E. Grey à C. Spring Rice du 30 juillet 1909 ; et FO 371/745/31501, remarque manuscrite d'E. Grey au verso du bordereau de réception (21 août 1909).

72 Folke Lindberg, op. cit., p. 176.

73 FO 371/745/16381, remarque manuscrite d'E. Grey au verso du bordereau de réception ( $1^{\text {er }}$ mai 1909).

74 Alec Tweedie, Through Finland in Carts, Londres, A. and C. Black, 1897.

75 Alexander MacCallum-Scott, Through Finland to St Petersburg, Londres, Grant Richards, 1908.

76 Rosalind Travers, Letters from Finland (1908-1909), Londres, Kegan Paul, 1911.

77 Philippe Blanc, «Les voyageurs britanniques dans les régions baltiques entre $\mathrm{XIX}^{\mathrm{e}}$ et $\mathrm{XX}^{\mathrm{e}}$ siècle : une perception fantasmée mais évolutive. Le cas de la Finlande ", Revue d'histoire nordique, no 18, 2014, p. 173-197. 
Mais dépassant le cercle restreint de ces quelques voyageurs, on assiste, en Grande-Bretagne comme en France ${ }^{78}$ et en Allemagne, à une véritable offensive médiatique, dirait-on aujourd'hui, en vue de promouvoir la spécificité de l'identité nationale finlandaise. À la source de cette offensive, on trouve toujours des militants de gauche, comme le journaliste Samuel G. Hobson ${ }^{79}$ ou Henry M. Hyndman, époux de Rosalind Travers et cofondateur de la Social Democratic Federation et du British Socialist Party ${ }^{80}$. Ces derniers épaulent un certain nombre de personnalités finlandaises de grande envergure, hommes politiques, intellectuels, artistes, devenus, pour l'occasion, de véritables commis voyageurs de la culture finlandaise, à l'enseigne de Leo Mechelin (dès 1890), du Pr Julio $\mathrm{N}$. Reuter, qui recrute Mrs Alec Tweedie ${ }^{81}$ et de l'anthropologue et philosophe Edvard Westermarck ${ }^{82}$.

C'est la campagne de russification menée en Finlande qui déclenche la contrecampagne des Finlandais en Occident, dans le but de sensibiliser l'opinion à la cause finlandaise. Et très vite, la presse britannique - dès les années 1890 - à travers des articles écrits soit par des Finlandais, soit par des journalistes sympathisants ${ }^{83}, s^{\prime}$ empare du sujet, et $s^{\prime}$ en fait l'écho auprès du public britannique sur le thème de la civilisation luttant contre les barbares, la liberté contre l'autocratie ${ }^{84}$.

Au final, le lobbying des Finlandais s'avère très payant puisque l'on voit apparaître dès 1899, dans la presse britannique, le concept de la Finlande en tant qu'État tampon entre la Scandinavie et la Russie ${ }^{85}$, et méritant, à ce titre, sa place pleine et entière parmi les pays occidentaux.

C'est le contraste entre l'état de l'opinion, au moins libérale et de gauche, et la realpolitik du gouvernement britannique, qui démontre, encore une fois, l'évolution dans la manière dont le Foreign Office traite à présent avec la Russie sur cette frontière baltique.

Déjà, au tournant du siècle, à mesure que l'on s'achemine vers une entente avec la Russie, la Finlande n'a même plus vraiment d'intérêt stratégique en tant que telle pour la Grande-Bretagne ${ }^{86}$. Toutefois, il n'en reste pas moins vrai, une

78 Sur l'activité et l'influence des élites finlandaises en France, lire Louis Clerc, « Perceptions croisées : Leo Mechelin et les réseaux finlandais en France (1880-1914) ", Cahiers de Fare, no 6, 2015, p. 133-147.

79 George Maude, «Finland in Anglo-Russian Diplomatic Relations, 1899-1910 », op. cit., p. 577.

80 Markku Ruotsila, Churchill and Finland. A Study in Anticommunism and Geopolitics, Londres New York, Routledge, 2005, p. 12.

81 George Maude, Finland and Britain. 1854-1914, op. cit., p. 199 et 203.

82 Ibid., p. 190 et 195.

83 À l'exemple du journaliste E.B. Lanin (alias E.J. Dillon) dans la Fortnightly Review, en janvier 1891 (ibid., p. 168, 169, 202 et 205).

84 Ibid., p. 202-205; et Juhani Paasivirta, op. cit., p. 199.

85 George Maude, Finland and Britain. 1854-1914, op. cit., p. 205.

86 George Maude, «Finland in Anglo-Russian Diplomatic Relations, 1899-1910 », op. cit., p. 558-561. 
fois l'entente de 1907 signée, qu'Edward Grey, devant les agissements russes en Finlande, et surtout leurs répercussions dans la presse, écrit en février 1908 à Arthur Nicolson, qu'il est " préoccupé " par la "politique réactionnaire " russe, et que «si la Finlande est maltraitée [...] l'opinion publique [britannique] sera très remontée ${ }^{87}$. Mais ce commentaire est moins l'expression d'une franche désapprobation de la politique russe que celle d'une irritation face à ce qu'il considère être une attitude irréfléchie de la part du gouvernement de Saint-Pétersbourg, comme dans le cas des îles Åland. Ainsi prévient-il le successeur d'Alexandre Izvolski, Sergei Dmitrievich Sazonov (1910-1916), de ne pas trop user de la manière forte pour affirmer l'autorité ou la présence russes en Perse comme en Finlande, à cause du danger d' "indisposer " le public britannique, dont une partie semble prompte à critiquer la convention de $1907^{88}$.

Il faut dire que la manière dont les principaux hauts fonctionnaires britanniques, connaisseurs de la Russie, rendent compte des événements n’est certainement pas sans influence sur la perception que peut avoir le ministre des Affaires étrangères quant au mouvement de russification en Finlande. Nous avons parlé des sympathies russes de l'ambassadeur Arthur Nicolson, mais on peut en dire au moins autant de son prédécesseur à Saint-Pétersbourg, Charles Hardinge, qui, en tant que sous-secrétaire d'État permanent au Foreign Office, occupe un poste clé dans le processus d'acheminement des informations vers le sommet du ministère britannique des Affaires étrangères. Les points de vue de ces deux personnages sont donc extrêmement importants et convergent, au moment du battage médiatique sur la Finlande, dans le sens d'une franche sympathie pour la politique finlandaise du Premier ministre Piotr Stolypine (1906-1911), Charles Hardinge allant jusqu’à considérer que les Finlandais feraient mieux de se soumettre ${ }^{89}$. La correspondance d'Arthur Nicolson à l'intention de son ministre de tutelle, quant à elle, bien qu'un peu moins sévère pour les Finlandais, parle d'elle-même. Si celui-ci admet le "bien-fondé » des " craintes du public finlandais " ${ }^{90}$, ainsi que leurs "revendications légitimes ", il met plutôt en avant le souci très compréhensible des Russes face à la proximité de la Finlande par rapport à la capitale de l'Empire, justifiant la politique de russification ${ }^{91}$.

Hormis ses inclinaisons personnelles et professionnelles, la compréhension dont fait montre Arthur Nicolson pour la politique de russification de la Finlande est aussi en partie due à la crainte de voir la Suède se précipiter un jour dans les bras de l'Allemagne, déjà échaudée par la question des îles Åland, si l'on fait trop

87 Lettre privée d'E. Grey à A. Nicolson du 24 février 1908, doc. no 550, in G.P. Gooch et H. Temperley (dir.), vol. IV, op. cit., p. 616-617.

88 Eino Lyytinen, op. cit., p. 51.

89 Ibid., p. 46.

90 FO 881/9317/30, lettre no 97 d'A. Nicolson à E. Grey du 22 février 1908.

91 FO 881/9728/1, lettre no 672 d'A. Nicolson à E. Grey du 23 décembre 1909. 
de cas - trop de publicité, pourrait-on dire - des brutalités russes, y compris visà-vis de populations svécophones ${ }^{92}$. Cela fait d'ailleurs écho à une autre crainte du Foreign Office, étayée par ce que dit Cecil Spring Rice de l'autre côté du golfe de Botnie, qui explique à Edward Grey que « si la Russie devait exercer un contrôle direct et absolu du gouvernement finlandais, l'État tampon [finlandais, relativement autonome,] disparaîtrait, et la Suède entrerait immédiatement en contact direct avec son voisin tant redouté $»^{93}$. Après la question d'Åland, on voit qu'en 1909 les sujets d'irritation ne manquent pas pour un Edward Grey qui fait tout son possible pour tenter de canaliser son nouvel ami russe, considérant que les enjeux stratégiques globaux ne doivent pas être éclipsés par des problèmes (frontaliers) locaux, auxquels Londres est, somme toute, peu sensible.

Dans la même optique, Arthur Nicolson considère que, de toute façon, la question finlandaise ne doit pas venir perturber le rapprochement anglo-russe, dont il est, rappelons-le, l'un des artisans. Il arrive même à convaincre Edward Grey « que toute critique du gouvernement russe jetterait la Russie dans les bras de l'Allemagne $"{ }^{94}$. Au final, ce discours s'avère efficace puisque le ministre des Affaires étrangères se range à cet avis, d'autant plus que celui-ci veut éviter à la fois l'isolement et l'implication dans une guerre européenne. Il reste donc « déterminé à sauvegarder l'entente avec la Russie malgré toutes les critiques ${ }^{95}$.

Edward Grey intervient même en personne, de près ou de loin, dans ce que l'on peut appeler une coopération passive avec la Russie, puisqu'il s'agit de ne pas gêner sa politique intérieure, même si cette politique peut mettre mal à l'aise le gouvernement d'un pays dans lequel l'opinion publique s'exprime librement. Ainsi, lorsqu'une délégation parlementaire, conduite par Mr Abraham, vient présenter à la Douma, en mai 1910, un mémorandum concernant l'incorporation de la Finlande dans l'Empire russe, signé par 163 des 670 membres de la Chambre des communes - " une action inopportune et mal avisée ", selon Arthur Nicolson ${ }^{96}$-, Edward Grey s'oppose à la démarche, car il s'agit d'une ingérence dans les affaires intérieures russes ${ }^{97}$. De même, lorsqu'une délégation de personnalités politiques britanniques vient visiter Saint-Pétersbourg en février 1912, on remarque, en Finlande, que celle-ci a été soigneusement expurgée de "tous les amis de la Finlande $»^{98}$.

92 FO 371/725/546, communication d'A. Nicolson à E. Grey du 6 octobre 1909, citée par George Maude, «Finland in Anglo-Russian Diplomatic Relations, 1899-1910», op. cit., p. 574.

93 FO 371/745/16381, dépêche de C. Spring Rice (Stockholm) à E. Grey du 27 avril 1909.

94 Eino Lyytinen, op. cit., p. 45.

95 Ibid., p. 51.

96 FO 881/9728/56, lettre no 239 d'A. Nicolson à E. Grey du 19 mai 1910.

97 Eino Lyytinen, op. cit., p. 48.

98 FO 881/10295/5, lettre n 37 de G. Buchanan à E. Grey du 3 février 1912. 
Autrement dit, pour résumer d'une manière un peu lapidaire le sentiment des diplomates britanniques, les Finlandais devraient se montrer beaucoup plus compréhensifs vis-à-vis des intérêts de la politique étrangère britannique! Or, précisément, les relations anglo-finlandaises restent assujetties à la politique russe de la Grande-Bretagne ${ }^{99}$.

\section{Conclusion}

La vision de la « frontière » qui semble prévaloir au travers des relations anglorusses durant cette période de transition, qui fait progressivement passer l'Europe $\mathrm{du} \mathrm{XIX}^{\mathrm{e}}$ vers le $\mathrm{XX}^{\mathrm{e}}$ siècle, nous semble découler de deux types de considérations qui se superposent. La logique géostratégique est, bien entendu, primordiale, tant sont nombreux les motifs de confrontation dans la région. Sur ce plan-là, la Baltique orientale constitue clairement, non pas une zone d'intérêt centrale pour la Grande-Bretagne, mais la périphérie européenne septentrionale de sa sphère d'influence. Parallèlement, la mer Baltique, d'une manière générale, reste un théâtre essentiel de relations économiques importantes et plus constantes, qui coexistent parfaitement même dans les moments de tensions politiques. Aussi, peut-être précisément à cause de la dimension périphérique de la Baltique, les deux logiques - géostratégique et économique - peuvent-elles, dans une certaine mesure, fonctionner indépendamment l'une de l'autre.

On voit d'abord que les bouleversements stratégiques internationaux n'affectent pas forcément la situation économique, et le fait est que les flux économiques entre le Royaume-Uni et la Baltique ne cessent de progresser sur toute la période choisie ici, surtout sur le plan des importations britanniques ${ }^{100}$. Il arrive même parfois que l'on sacrifie certains intérêts économiques privés sur l'autel de la coopération anglo-russe, puisque sont balayées du revers de la main les protestations des chambres de commerce britanniques qui craignent que l'incorporation complète de la Finlande dans l'Empire ne conduise à une augmentation spectaculaire des tarifs douaniers ${ }^{101}$. Inversement, au plus fort des événements de Crimée (début 1855), malgré l'état de guerre effectif, le gouvernement de Lord Aberdeen s'empresse de ne rien faire qui empêcherait complètement "l'existence d'un commerce avec la Russie en temps de guerre ", pratiqué avec beaucoup de zèle par les marchands britanniques (sur des navires étrangers), afin que ces derniers conservent « leur suprématie dans le commerce russe ${ }^{102}$.

99 Eino Lyytinen, op. cit., p. 26.

100 En valeurs absolues approximatives, les importations britanniques depuis l'ensemble baltique (hors Allemagne) passent de 15450000 livres sur la période 1856-1860, à 37320000 livres pour 1885-1889, et à 55740000 livres pour 1906-1910. Philippe Blanc, La Grande-Bretagne, le Nord et la Baltique..., op. cit., p. 216 et 358.

101 Eino Lyytinen, op. cit., p. 48-49.

102 Bernard Semmel, Liberalism and Naval Strategy, Boston, Allen \& Unwin, 1986, p. 54-56. 
Une fois les fondamentaux économiques sauvegardés, ceux-ci devant d'ailleurs être envisagés sur le plan mondial plutôt que régional, comme le dit Edward Grey lui-même, "les ministres des Affaires étrangères britanniques [sont] guidés par ce qui leur semble être l'intérêt immédiat du pays, sans faire de calculs élaborés quant à l'avenir... ${ }^{103}$. On comprend mieux, dès lors, les louvoiements et revirements constatés durant la période qui nous intéresse.

Concernant la Baltique, ce sont donc des considérations de niveau européen, voire asiatique, qui président à la conduite que l'on adopte vis-à-vis de la « ligne de circonvallation » issue de la guerre de Crimée. Les contemporains le voient très bien, puisqu'en 1907, année qui constitue un tournant avec l'entente anglo-russe, un conseil ministériel suédois décrit la Grande-Bretagne comme une "puissance éloignée, dont les intérêts dans la région nordique sont habituellement subordonnés aux exigences de sa politique étrangère générale " ${ }^{104}$. C'est aussi en ce sens-là que, vue depuis Londres, la zone baltique n’est que périphérique.

Au tournant de 1906-1907, précisément, pour la Grande-Bretagne, le vieil ennemi russe est devenu au moins un partenaire respectable malgré son autocratie archaïque. Ainsi, localement, on est passé de relations fondées sur une confrontation à une forme de coopération, plus ou moins passive ; et le traitement de la "frontière » baltique s'en ressent, car il s'agit de préserver l'entente avec la Russie en faisant tout ce qui est raisonnablement possible pour que son gouvernement ait les mains libres sur son pourtour baltique. On accepte même, dans les mois qui précèdent le conflit mondial, d'aller bien plus loin dans la coopération locale avec la Russie, puisque Edward Grey écrit, en mai 1914, que " nos forces navales seraient mieux utilisées en dehors de la Baltique, et la Russie pourrait utiliser ses propres forces navales du mieux possible à l'intérieur de la Baltique » ${ }^{105}$, alors qu'il s'est agi, pendant des décennies, d'éviter à tout prix la formation d'une mare russicum au Nord de l'Europe !

Toutefois, preuve supplémentaire que la politique baltique de la GrandeBretagne n'a pas fini d'obéir à des logiques stratégiques différentes, la nouvelle " ligne de coopération " anglo-russe redeviendra un lieu de confrontation après le conflit mondial lorsqu'il faudra lutter contre l'ennemi bolchevique pendant la guerre civile russe $(1918-1921)^{106}$.

103 Viscount Grey of Fallodon, Twenty-five Years. 1892-1916, vol. I, Londres, Hodder \& Stoughton, 1925 , p. 6.

104 Minutes d'un conseil ministériel suédois du 23 décembre 1907, citées par Wilhelm M. Carlgren, op. cit., p. 30.

105 Communication d'E. Grey à l'ambassadeur F. Bertie (Paris) du $1^{\text {er }}$ mai 1914, citée par David W. Sweet, op. cit., p. 484.

106 Lire à ce sujet Olavi Hovi, The Baltic Area in British Policy. 1918-1921, Helsinki, Finnish Historical Society, 1980, 2 vol. ; et Markku Ruotsila, op. cit. 